\title{
EL IMPACTO DE LAS NUEVAS FORMAS ORGANIZATIVAS PROPIAS DE LA ECONOMÍA BASADA EN MEDIOS TECNOLÓGICOS EN EL MUNDO DEL TRABAJO
}

THE IMPACT OF THE NEW ORGANIZATIONAL FORMS OF THE ECONOMY BASED ON TECHNOLOGICAL MEANS IN THE WORLD OF WORK

\author{
Cecilia Calderón Paredes*
}

Garrigues

The Congress of the Republic of Peru has received two bills with the purpose of including legal relationships composed of digital platforms, the companies involved and those who provide services for these platforms within the scope of Labor Law Regulations as a way to prevent them from being improperly excluded from such application of the law.

This article explores the concept of on-demand economy and the economy's new organizational methods that has been propelled by technological advances in the world of work and examines them through the review of international jurisprudence.

KEY WORDS: on-demand economy; digital platforms; work relationship; subordination.
El Congreso de la República del Perú ha recibido dos proyectos de ley con la finalidad de incluir en el ámbito de la normativa laboral, la relación jurídica conformada por plataformas digitales, las empresas implicadas y aquellos que brindan servicios para estas plataformas como forma impedir que estas indebidamente sean excluidas del ámbito del Derecho del Trabajo.

El presente artículo explora el término on-demand economy, las nuevas formas organizativas de la economía en base a innovaciones tecnológicas en el mundo del trabajo y las examina mediante la revisión de jurisprudencia internacional.

PALABRAS CLAVE: on-demand economy; plataformas digitales; relación laboral; subordinación.

\footnotetext{
Abogada. Magíster en Derecho de las Transacciones Internacionales por el Centro de Estudios Garrigues y la Universidad Antonio de Nebrija, España. Ha seguido estudios de especialización en la Pontificia Universidad Católica del Perú y en la Universidad de Castilla-La Mancha. Asociada Senior del Estudio Garrigues. Contacto: cecilia.calderón@ garrigues.com.
}

Nota de Editor: El presente artículo fue recibido por el Consejo Editorial de THËMIS-Revista de Derecho el 01 de junio de 2019 y aceptado por el mismo el 26 de julio de 2019. 


\section{INTRODUCCIÓN}

El desarrollo de la tecnología y su proliferación en las necesidades cotidianas han generado una economía sustentada en ella. Una muestra saltante de este fenómeno es que, en nuestros tiempos, muchas de las relaciones jurídicas en las cuales nos desenvolvemos se sustentan en la denominada on-demand economy ${ }^{1}$, un nuevo modelo económico que trastoca el paradigma de la producción y el consumo en masa y se basa en la revalorización del uso o la posesión de los bienes por encima de la propiedad (Gauthier Guazzoni, 2016) la cual ha echado mano del uso de las nuevas tecnologías para su expansión. A pesar de su creciente y evidente relevancia, muy poco se ha dicho sobre el impacto que esta genera en sus principales actores: los proveedores de servicios.

La discusión y el análisis del tipo de prestación provista bajo estas nuevas formas de organización se ha llevado a cabo principalmente en el mundo anglosajón y en la comunidad europea continental, donde lo primero que ha salido a relucir es una presunta competencia desleal que se estaría generando presuntamente en el mercado a consecuencia de su aparición².

En nuestro país, aunque no se trata de empresas cuyas actividades sean de aparición reciente, las plataformas digitales se convirtieron en el foco de atención a raíz de que, a mediados del año 2017, se presentaron en medios informativos diversas denuncias por la comisión de delitos tales como hurto, robo o, inclusive, actos de violencia sexual, lo cuales apuntaban a quienes proveen servicios a través de plataformas digitales o apps (en adelante, la app, las apps o las plataformas), mediante los que ofrecían sus servicios de taxi.

Lo cierto es que, a consecuencia de estos hechos, la sociedad empezó a preguntarse quién se hacía responsable por los daños que pudieran generar los conductores a los cuales se contactaba a través de las plataformas. Frente a este problema comenzó a evidenciarse que, contrariamente a lo que muchos esperaban, en realidad las personas que ejecutaban los servicios a través de las apps lo hacían de forma independiente a estas, toda vez que los titulares de las plataformas no mantenían con aquellos algún vínculo que generase que actúen en su nombre o las representasen.

Esta materia ya ha generado una reacción a nivel legislativo, si bien aún no se ha convenido en una legalidad concreta. En efecto, en abril del presente año, el Congreso de la República ha recibido dos proyectos de ley promovidos por distintos grupos parlamentarios con una idéntica finalidad: que en el repertorio de la variada normativa laboral se incluya la relación jurídica que vincula a quienes prestan los servicios ofertados en una app con el titular de la misma.

El presente ensayo tiene como objetivo analizar el impacto del modelo organizativo de la economía generada por el uso de plataformas en las relaciones establecidas entre las personas que brindan los servicios ofrecidos en ellas y las compañías titulares de la tecnología, para lo cual además de considerar la legislación y doctrina nacional reflexionaremos en función a doctrina y pronunciamientos extranjeros sobre la materia.

\section{LAS CARACTERÍSTICAS DE LA ON-DEMAND ECONOMY Y SU IMPACTO EN EL ESTABLECI- MIENTO DE UNA RELACIÓN LABORAL}

\section{A. Nuevas formas de organización empresa- rial de la mano del creciente protagonismo de la tecnología como principal activo del negocio}

Como habíamos adelantado, la tecnología ha alcanzado, desde hace ya algunos años, un alto nivel de contribución a la vida diaria: ya no es más un apoyo complementario en el desarrollo de relaciones productivas (por ejemplo, a través del uso de una computadora, programas de cálculo, Internet y correos electrónicos corporativos, entre otros), sino que se trata de la piedra angular de nuevos esquemas empresariales.

Así, en torno a los avances tecnológicos y a las amplias posibilidades que se abren a partir de ellos, se ha creado una nueva estructura de relaciones económicas que se apoya en la especialización de los servicios y en la valorización de la tecnología como un medio de conexión. Inclusive, existen quienes afirman que el principal activo de estas economías

Para mayor detalle, ver a Gauthier Guazzoni, G. (2016).

2 El uso de la tecnología ha proliferado, por ejemplo, en los aplicativos que facilitan el transporte de personas y en vista de que en muchas jurisdicciones éste pertenece a un sector regulado, se alega que mientras no se regule la explotación de dichos aplicativos se podría estar permitiendo la generación de competencia desleal: quienes operan a haciendo uso de plataformas digitales no están obligados a tramitar licencias o autorizaciones previas ni a contratar seguros contra accidentes o terceros -lo que sí deben hacer quienes operan en el sector-, algo que abarata sus costos y, por ende, la tarifa de sus servicios. 
es el algoritmo a partir del cual funcionan. En este sentido, se afirma que "en el futuro, las empresas se valorarán no solo por sus grandes bases de datos, sino por los algoritmos que transformen esos datos en acciones de impacto para los clientes..." (Gauthier Guazzoni, 2016, p. 3).

La on-demand economy es, pues, un modelo de negocio desarrollado aprovechando las ventajas del internet y que se basa en plataformas virtuales que albergan grandes grupos de proveedores que ofrecen sus servicios de manera amplia, a un mercado abierto que desconocen, quedando a la espera de que sus respectivas apps les asignen algún encargo o que algún interesado solicite de forma expresa sus servicios. La clave del éxito de este tipo de negocio -desde el punto de vista del titular de la plataforma- radica en contar con un gran número de personas que puedan proporcionar el servicio requerido por un tercero pues así su negocio garantiza una oferta suficiente que permite cubrir o satisfacer la demanda existente por los usuarios de la app en cualquier momento. Al respecto, es importante tener presente que el comportamiento de los consumidores ha evolucionado de la mano de la tecnología, el internet y el acceso inmediato a las funcionalidades en línea, generándose una creciente necesidad de experiencias de consumo rápidas, simples y eficientes, características que, al ser cubiertas por las apps, garantizarán una mayor aceptación y éxito de la empresa en el mercado.

Tal y como lo plantean las empresas titulares, sus plataformas proporcionan una infraestructura invisible que conecta la oferta y la demanda existentes en el mercado, facilitando la interacción de las partes de una transacción: los proveedores de servicios y aquellos que los necesitan (i.e. los clientes). Así, este modelo de negocio se construye a partir de tres elementos: (i) el público o el consumidor, compuesto por personas que requieren la prestación de un servicio; (ii) las personas que prestan los servicios de forma individual y directa; y, (iii) las compañías que han desarrollado las plataformas donde se ofrece el servicio, las que se consideran a sí mismas como el medio que sirve para vincular la oferta y la demanda, servicio por el que reciben un porcentaje de las transacciones que se acuerden a través de ellas.

Ahora bien, desde nuestra perspectiva, aunque en la on-demand economy las empresas alegan no proporcionar directamente el servicio requerido por los consumidores, sino ser simples intermediarios entre proveedores y usuarios, lo cierto es que una amplia zona gris puede generarse como consecuencia de cuál sea la forma de relacionarse que tenga la empresa titular de la app con el proveedor que hace uso de ella. En efecto, en la mayoría de los casos, las empresas titulares de las plataformas ejercen algún tipo o grado de injerencia respecto del comportamiento de los proveedores pues, como es evidente, el éxito de su plataforma en el mercado $-y$, por tanto, los ingresos de la empresa- dependerá del nivel de satisfacción que manifiesten los usuarios, la que dependerá de las actividades de los proveedores.

Por lo dicho, aun cuando se presentan a sí mismas como simples intermediarias, la lógica económica nos lleva a entender e, inclusive, considerar razonable, que exista un interés de parte del titular de la plataforma en la manera en la cual el servicio se vaya a prestar toda vez que, si los proveedores que la app contacta con los usuarios no ejecutan sus actividades de manera satisfactoria para ellos, estos últimos no solo no seguirán utilizando la app sino que, además, es probable que también desalienten a otros a hacerlo, reduciendo o limitando su mercado y, por lo tanto, afectando gravemente a la empresa.

Por lo dicho, en un próximo apartado analizaremos cómo es que en el marco de lo que parecen ser simples relaciones comerciales pueden asomarse elementos o indicios que nos llevan a considerar la posibilidad de que se configuren vínculos de naturaleza laboral.

B. ¿Por qué es necesario considerar la aplicación del Derecho Laboral en vínculos que inicialmente pretenden regular relaciones civiles?

Históricamente, el uso de la tecnología para el desarrollo de negocios ha generado muchos desafíos para el Derecho del Trabajo. Aunque, al principio, el temor principal era que las máquinas reemplazaran a la fuerza laboral del hombre, el impacto de las tecnologías en las relaciones laborales no es un problema que hubiere quedado desfasado. De hecho, en las últimas décadas, la incorporación de mayores y más sofisticados avances tecnológicos -e.g. el uso de cámaras, GPS y teléfonos inteligentes, entre otros- ha generado cuestionamientos sobre la forma en que las personas son monitoreadas, así como si el uso de estos instrumentos perturba o no el derecho a la privacidad que tienen las personas en el contexto de la relación laboral.

Concentrándonos en los problemas que podrían asociarse con la on-demand economy, recordamos que una de las razones que históricamente ha determinado la necesidad de una protección específica para quienes prestan servicios dependientes es la desigualdad existente entre los mismos y los empleadores -ya sean personas naturales o jurídicas-. Esta desigualdad se experimenta, 
por ejemplo, cuando se evalúa el poder de negociación de ambas partes, experimentándose tangiblemente al momento de plasmar los términos y condiciones que rigen el vínculo.

No obstante, la desigualdad es una característica que se encuentra en distintos ámbitos de una sociedad, por lo que conviene preguntarnos, ¿por qué el Derecho del Trabajo -y, por tanto, la problemática que busca evitar- debiera aplicarse a los vínculos jurídicos que se desarrollan en la ondemand economy?

Revisando la jurisprudencia y las opiniones doctrinales que se han desprendido de ella, encontramos que quienes critican el negocio que se genera en base a la explotación de plataformas lo hacen alegando que éstas estarían generando nuevas formas de empleo precario pues, utilizando terminología que apunta más a la formación de un vínculo autónomo, pretenderían disfrazar nuevas formas de dependencia o subordinación.

Al respecto, y como sucede muchas veces en el ámbito del Derecho del Trabajo, a nuestro entender la existencia o no de un vínculo de naturaleza laboral en el desarrollo de un negocio desarrollado a través de una app dependerá de las circunstancias y características que lo rodean, no pudiendo alegarse de forma general que en todos los modelos económicos que implican una app o un algoritmo exista un régimen de subordinación amparable desde el Derecho del Trabajo: se genera, pues, la necesidad de un análisis casuístico.

Así, aunque no todas las empresas de la on-demand economy celebran acuerdos con los proveedores bajo las mismas reglas o condiciones, cierto es que, a nivel comparado, en algunos casos se ha identificado que tanto las facultades o derechos que la empresa titular de la app se reserva se manifiestan en la forma cómo se comporta en relación con el proveedor generando una distorsión o desnaturalización del contrato de servicios independientes y convirtiéndolo así en un contrato de trabajo.

Resulta importante, entonces, tener presente que los problemas que pueden surgir a partir de una calificación indebida de prestadores de servicios subordinados como independientes son muchos y muy complejos. Por ejemplo, cuando se procede a calificar un servicio como independiente, el proveedor del mismo no necesita atender las reglas aplicables a las horas de trabajo máximo y, por lo tanto, podría terminar superando cualquier límite legal; de igual forma, podría darse el caso de que las tarifas acordadas con los proveedores sean muy reducidas y que, debido a ello, éstos se vean en la necesidad de prestar servicios en mayor número de horas para generar las ganancias diarias que necesitan.

En realidad, la indebida calificación de personal dependiente como independiente es un problema social cuyo impacto es, inclusive, mayor que el económico pues, por ejemplo, la regulación sobre salarios mínimos prevista como parte del régimen de actividad laboral no solo tiene como objetivo proteger a los trabajadores sino al mercado en general, especialmente considerando que la existencia de salarios bajos impide la capacidad de adquisición de las personas, lo que limita a su vez la economía del país. Otro problema que podría aumentar en función del crowdsourcing es el desarrollo del trabajo infantil (Todolí, 2015, p. 24) ya que los que prestan servicios a través de plataformas -especialmente en el caso de los servicios ejecutados en línea- podrían ser menores de edad que lo hacen durante su tiempo libre o, incluso, como una forma de vida, algo que no solo vulnera los compromisos asumidos por los Estados ante la Organización Internacional del Trabajo (OIT) sino que, además, precariza las condiciones de vida de la población.

Debido a lo mencionado, y dado que entendemos que la on-demand economy ha creado ocupación e ingresos en países con distintas realidades -desde economías privilegiadas como la británica o la estadounidense, hasta economías menos favorecidas como los países de Latinoamérica o, inclusive, la española ${ }^{3}$ que actualmente ocupa el segundo lugar en desocupación en el continente europeo, duplicando el porcentaje de personas no ocupadas de la región siendo sólo superada por Gre$\mathrm{cia}^{4}-$, consideramos que lo más adecuado será no satanizar su existencia sino más bien analizar sus componentes y las prerrogativas que se reservan las empresas, buscando evitar que se simiente en vínculos independientes desnaturalizados.

Conforme a ello, a continuación, analizaremos brevemente los elementos característicos de la relación laboral para luego determinar qué aspectos deberán tomarse en cuenta al momento de analizar su alegada existencia en el marco de la prestación de servicios que se genera utilizando las apps.

3 De acuerdo con información del Instituto Nacional de Estadística de España (INE) que corresponde al Trimestre 2/2019, el porcentaje de la población española en paro asciende a 14.7\% Ver Instituto Nacional de Estadística (2019).

4 Véase la información difundida por el portal El Mundo (2019) que indica que España y Grecia lideran el desempleo en la Unión Europea, alcanzando tasas que duplican la media. Ver a Urrutia, C. (2019). 


\section{Elementos de la relación laboral observa- dos en el contexto de la on-demand eco- nomy}

De conformidad con lo dispuesto en el artículo 4 del Decreto Supremo 003-97-TR, TUO de la Ley de Productividad y Competitividad Laboral, toda relación laboral presenta tres elementos esenciales, a saber, (i) la prestación personal de servicios; (ii) la remuneración; y, (iii) la subordinación.

Así, como sostiene Neves:

[...] la doctrina concuerda en que esos elementos son tres: prestación personal, subordinación y remuneración. La jurisprudencia nacional lo estableció así desde ya hace un buen tiempo [...] Para que nos encontremos ante una relación laboral, entonces, los mencionados elementos deben presentarse en forma conjunta. Si alguno de ellos falta, la relación jurídica tendrá otra naturaleza. Por ejemplo, si hay una prestación personal y retribuida, pero autónoma [...]" (2012, p. 16) [El énfasis es nuestro].

Ahora bien, a nuestro parecer, la subordinación es el elemento que distingue al contrato de trabajo de los contratos civiles de locación de servicios $y$, por ende, es el que se debe evitar en caso se pretenda establecer vínculos jurídicos ajenos a los efectos regulados por el Derecho del Trabajo. ¿Por qué nos permitimos afirmar ello? Porque en los vínculos de naturaleza mercantil suele existir una retribución por el servicio prestado -a menos que el mismo se ejecute a título gratuito-e, inclusive, existen circunstancias en las que la prestación personal del servicio es requerida, respondiendo, por ejemplo, a la especialidad del mismo o al hecho de que las características del prestador fueron consideradas a efectos de la contratación -algo que no es ajeno, por ejemplo, a la prestación de servicios legales en las que en muchas ocasiones los clientes contratan de forma independiente a un abogado específico, atendiendo a su trayectoria, especialidad, reconocimiento en el medio, confianza, entre otros, lo que determina que dicha persona no pueda ser sustituida por otro profesional del mismo rubro, así se trate de uno que forma parte del staff de la misma firma y que, inclusive, esté igual o mejor calificado para el servicio en concreto.

En el mismo sentido, el Tribunal Constitucional peruano ha señalado en la sentencia recaída en el Expediente 9197-2006-AA-TC, que la diferencia entre una relación de naturaleza laboral (contrato de trabajo) y una civil (contrato de locación de servicios) consiste en que en esta última no se presenta el elemento de dependencia o sujeción. Así, ha señalado que:

[...] el contrato de locación de servicios ha sido definido en el artículo 1764 del Código Civil como aquél acuerdo de voluntades por el cual "el locador se obliga, sin estar subordinado al comitente, a prestarle sus servicios por cierto tiempo o para un trabajo determinado, a cambio de una retribución" [por lo que] es evidente que, de la definición dada por el Código Civil, se desprende que lo que caracteriza a este contrato es la independencia del locador frente al comitente en la prestación de sus servicios, siendo este el elemento diferenciador entre ambos contratos [...] [El énfasis es nuestro].

Luego, al ser la subordinación el elemento central que define la existencia de un vínculo laboral, conviene preguntarnos en qué consiste, pues la única forma en que un vínculo jurídico pueda extraerse del ámbito laboral será evitar que se ejecute en un contexto de subordinación. Así, como señala Neves:

[...] la subordinación es un vínculo jurídico entre el deudor y el acreedor de trabajo, en virtud del cual el primero le ofrece su actividad al segundo y le confiere el poder de conducirla. Sujeción, de un lado, y dirección del otro, son los dos aspectos centrales del concepto [...] (2012, p. 36) [El énfasis es nuestro].

Desde nuestra perspectiva, siempre que se pueda identificar que una de las partes ostenta el poder de dirigir, fiscalizar y sancionar a la otra, nos encontraremos ante una relación de sujeción o subordinación entre ellas, característica que activa la aplicación de todo el régimen de protección laboral.

A nivel internacional, la Comisión Europea 5 (en adelante, la Comisión) aprobó en junio de 2017 una comunicación Para el establecimiento de una Agenda europea para la economía colaborativa ${ }^{6}$, estudio en el que se analiza el establecimiento de vínculos de naturaleza laboral en el contexto de la on-demand economy. Al respecto, al igual que la legislación y jurisprudencia nacional, la Comisión señala que el hecho de que exista un vínculo de naturaleza laboral debe determinarse en función de cada caso, considerando los hechos que caracterizan la relación existente entre la plataforma y el prestador de

5 Órgano ejecutivo de la Unión Europea, responsable de elaborar propuestas de nueva legislación europea y de aplicar las decisiones del Parlamento Europeo y el Consejo de la Unión Europea.

6 Para el texto de la citada comunicación en todas sus versiones ver EUR-Lex (s.f. b). 
servicios, basándose para el análisis en los siguientes tres criterios esenciales: (i) la existencia de una relación de subordinación; (ii) la naturaleza del servicio; y, (iii) la presencia de una remuneración.

Respecto al criterio de subordinación, la Comisión Europea indica que para que ésta se verifique, el proveedor del servicio deberá actuar bajo la dirección de la empresa propietaria de la plataforma, lo que de conformidad con lo establecido en la página 5 de la comunicación Reafirmación de la libre circulación de trabajadores: derechos y avances importantes significa que la empresa propietaria de la plataforma determine libre y unilateralmente la elección de la actividad, remuneración y condiciones de trabajo aplicables al servicio i.e. que el prestador no sea libre de elegir qué servicios ejecutará y cómo. Sobre el particular, es importante señalar que la Comisión, distinguiendo el ejercicio del derecho de la titularidad del mismo, ha indicado específicamente que "[...] la existencia de subordinación no depende necesariamente del ejercicio efectivo de la gestión o supervisión de manera continua [...]" (EUR-Lex s.f. b), lo que significa que el hecho de que la empresa ejerza o no subordinación no significará que tal derecho o facultad no le hubiera sido reservado.

En lo que se refiere a la naturaleza del servicio prestado, la Comisión ha establecido que el proveedor debe realizar "[...] una actividad de valor económico que sea real y efectiva, exceptuando las actividades que se lleven a cabo a una escala $\tan$ pequeña que puedan considerase meramente marginales y accesorias [...]" (EUR-Lex, s.f. a). Finalmente, sobre el criterio de remuneración, la Comisión ha expresado que se refiere a la necesidad de identificar una prestación de servicios que genere beneficios, señalando que cuando el proveedor se limita a recibir una suma que únicamente compensa o subsana los costos en los que hubiera incurrido por sus actividades, el requisito de tratarse de una actividad remunerada no se habría cumplido.

Encontramos, por tanto, que inclusive dentro del contexto de las economías colaborativas, lo relevante a efectos de determinar la aplicación o no del Derecho del Trabajo será identificar la reserva de poder de dirección en favor de la empresa.

\section{REVISIÓN DE LA JURISPRUDENCIA COM- PARADA: LOS CASOS UBER, GLOVO Y DE- LIVEROO}

Lejos de pronunciarnos sobre algún caso en particular, nuestro estudio se ha servido de jurispru- dencia extranjera vinculada al modelo de negocios basado en la explotación de plataformas con la intención de extraer los diferentes elementos encontrados en ellos que, a entender de los tribunales foráneos, apoyarían la necesidad de calificar el vínculo jurídico suscrito entre un prestador que ofrece sus servicios a través de una app con el titular de la misma como uno de naturaleza laboral.

\section{A. Pronunciamientos referidos a Uber}

Uber, empresa que pone a disposición de distintos usuarios una serie de conductores de vehículos particulares, es quizás una de las compañías que han sido más cuestionadas globalmente. Ello debido a que es una de las primeras empresas que presentó rotundo éxito en el modelo económico que proponía, lo que despertó interés en las jurisdicciones donde actuaba.

En el caso O'Connor v. Uber Technologies, con fecha 11 de marzo de 2015, el Tribunal de Distrito del Norte de California de los Estados Unidos determinó la existencia de una relación laboral entre las personas que prestaban sus servicios a través de la plataforma y la empresa. Esta decisión se apoya en la forma de organización del negocio en esa jurisdicción y en el espacio temporal, alegándose que, por cómo la empresa operaba frente a los usuarios, era posible determinar que la misma no se limitaba a la venta de software sino que comercializa viajes, hecho que la convertiría, en realidad, en una compañía de transporte que, sin embargo, utiliza "tecnología muy sofisticada" para lograr sus objetivos (Mercader, 2017, p. 8).

Por su parte, el Tribunal de Trabajo de Londres dictaminó el 26 de octubre de 2016 (caso Aslam v. Uber) que Uber utilizaba "un lenguaje retorcido" y una "terminología completamente nueva" que generaba cierto "escepticismo" al momento de evaluar su comportamiento empresarial. A nuestro parecer, la citada sentencia evidencia cierta suspicacia de la Corte que se reflejó en su análisis, llegando a incluir afirmaciones tan severas como que, en su opinión, es "ligeramente absurdo/descabellado" afirmar que Uber "[...] es un mosaico de 30,000 pequeñas empresas vinculadas por una "plataforma" común [considerando que] en cada caso, el "negocio individual" consiste en un hombre con un automóvil que busca ganarse la vida conduciéndolo [...]"7

A nuestro parecer, quizás en una realidad social y económica como la inglesa, pueda parecer descabellado afirmar que quienes prestan servicios de

\footnotetext{
Apartado 90 de la Sentencia de los Tribunales de Trabajo de Londres recaída en el expediente del caso 2202551/2015 seguido por Aslam, Farrar \& otros contra Uber B.V., Uber London Ltd. y Uber Britannia Ltd
} 
taxi con sus vehículos se consideren a sí mismos como independientes con un negocio propio. Pero en una realidad como la nuestra, en la que la falta de oferta de un empleo formal ha prevalecido por años, el hecho de que un bien como el vehículo personal sea la principal herramienta de trabajo y que se trate de un sector con una regulación de requisitos mínimos ha creado una conciencia generalizada respecto de que estas personas son sus propios jefes, pues disponen y se organizan a total voluntad. Por lo dicho, en este caso nos parecería inadecuado trasladar las conclusiones de la Corte inglesa a una realidad como la peruana, pues el contexto en el que se ha expedido difiere mucho de nuestras circunstancias.

Es relevante destacar que, de acuerdo a lo alegado por el citado Tribunal de Trabajo, el uso de algún elemento tecnológico que facilite la prestación de un servicio no convierte a la empresa que lo haga en una compañía de tecnología, sino que únicamente la moderniza o, inclusive, la hace más eficiente.

Ahora bien, al analizar los términos y condiciones que vinculan a las partes en virtud del Acuerdo de Servicios que se suscribe en aquella jurisdicción, el Tribunal encontró una serie de elementos que a su entender acreditarían la existencia de una relación laboral, pues la empresa titular de la app: (i) tiene un interés especial en quién rendirá los servicios ofrecidos en su plataforma (entrevista y selecciona a las personas que harán uso de ella como proveedores); (ii) dicta instrucciones a los proveedores para la prestación del servicio (por ejemplo, estableciendo la ruta que deben seguir los conductores); (iii) se reserva información clave del cliente que no comparte con los proveedores de servicios (por ejemplo, no revela el destino del usuario antes del inicio del viaje, así como tampoco le informa el número de la tarjeta de crédito utilizada para el pago); (iv) establece unilateralmente las tarifas del servicio, las que tienen como característica principal el ser no negociables; (v) impone condiciones y/o características respecto a la herramienta de trabajo (vehículo a utilizar); (vi) obliga y/o condiciona, indirectamente, el desempeño del trabajo (requiere que los conductores, bajo la posibilidad de ser sancionados, acepten un cierto número de viajes y no los cancelen una vez que hubieren sido aceptados); (vii) se comporta ante los usuarios de la app como si éstos fueran sus clientes (evaluando las quejas de los clientes); y, (viii) tiene una posición dominante en relación con el proveedor de servicio pues se reserva el derecho a modificar unilateralmente las condiciones que los vinculan; entre otros.

Por otro lado, con fecha 20 de diciembre de 2017, el Tribunal de Justicia de la Unión Europea emitió una sentencia en el caso C-434/15, en la cual se había solicitado una decisión preliminar referida a si la actividad que ejecuta Uber ofreciendo su plataforma califica o no como un servicio de transporte; pese a ello, el Tribunal de Justicia de la Unión Europea incluye entre los considerandos que componen su decisión una serie de afirmaciones que tienen un impacto en la determinación de la naturaleza de las relaciones que vinculan legalmente a los proveedores de servicios con la empresa, enumerando algunos elementos que implican el establecimiento de una relación laboral entre las partes.

Así, la decisión preliminar emitida por el Tribunal de Justicia de la Unión Europea señala que Uber ejercería una "influencia decisiva" sobre las condiciones bajo las cuales los conductores prestan sus servicios, determinando, al menos, la tarifa máxima, así como recolectando el dinero pagado por el usuario consumidor y pagando solo parte de ella al conductor del vehículo. De igual forma, el Tribunal de Justicia también señala que las consideraciones puestas en su conocimiento determinan que Uber ejerce control sobre la calidad de los vehículos en los que se prestarán los servicios ofertados en su app, así como sobre los conductores y su comportamiento, todo lo que puede resultar, en algunas circunstancias, en la exclusión de éstos de la app que comercializa la plataforma ${ }^{8}$.

\section{B. Pronunciamientos referidos a Glovo}

Glovo APP 23, SL (en adelante, Glovo) es una empresa fundada en el año 2014 en Barcelona que pone a disposición de distintos usuarios una serie de personas que la ayudan haciendo repartos; esta, a diferencia de Uber, ha recibido diferentes pronunciamientos en la justicia española, todos ellos a nivel de juzgado. Efectivamente, mientras algunos juzgados han calificado como un trabajador autónomo económicamente dependiente $(\text { trade })^{9}$ a quienes prestan servicios a través de la

$8 \quad$ Apartado 39 de la sentencia del Tribunal de Justicia de la Unión Europea (Corte de Luxemburgo), recaída sobre el Caso C-434/15 seguido por la Asociación de Taxistas Profesionales Elite contra Uber Systems Spain SL.

9 Concepto jurídico previsto en la Ley 20/2007, Estatuto del Trabajo Autónomo (2007), que regula a aquellos trabadores independientes que, pese a desempeñarse con autonomía funcional, desarrollan su actividad con una fuerte y casi exclusiva dependencia económica frente al empresario o cliente que los contrata -al menos $75 \%$ de sus ingresos dependen de él-. Se diferencian, pues, del llamado falso autónomo (i.e. de aquél trabajador cuyo vínculo laboral ha pretendido ser regulado como si se tratase de un independiente) en que no se trata de un trabajador por cuenta ajena cuyas actividades se integren en la organización empresarial, sino que gozan de autonomía funcional. 
app de Glovo, otros juzgados han reconocido la existencia de un vínculo de naturaleza laboral entre la empresa y los proveedores.

Así, por ejemplo, mediante Sentencia $284 / 2018^{10}$ el Juzgado de lo Social 39 de Madrid desestimó con fecha 3 de setiembre de 2018 la demanda formulada por despido en contra de la empresa Glovo. Al respecto, conviene señalar que el Juzgado parte de identificar como la principal actividad de la empresa

[...] el desarrollo y gestión de plataformas informáticas mediante las cuales, a través de una aplicación o página web, se permite a comercios locales [...] ofertar sus productos a través de la aplicación (APP) y, en su caso, si los consumidores finales así lo solicitan, intermediar en el transporte y entrega de los productos al cliente final $[\ldots]^{11}$.

Ahora bien, respecto de la existencia de un vínculo jurídico de naturaleza independiente, el juzgado valoró que, de acuerdo con los hechos probados en el proceso, en la relación jurídica que vinculaba a las partes no concurrían las notas definitorias de una relación laboral ${ }^{12}$, a saber:

a) Ajenidad del trabajo: Constituyen indicios comunes de la misma, la entrega o puesta a disposición del empresario por parte del trabajador de los productos elaborados o de los servicios realizados; la adopción por parte del empresario y no del trabajador de las decisiones concernientes a las relaciones de mercado o de las relaciones con el público, como fijación de precios o tarifas, selección de la clientela, indicación de personas a atender; el carácter fijo o periódico de la remuneración del trabajo; y el cálculo de la retribución o de los principales conceptos de la misma con arreglo a un criterio que guarde cierta proporción con la actividad prestada, sin el riesgo y sin el lucro especial que caracterizan la actividad del empresario o del libre ejercicio de las profesiones; $y$,

b) Dependencia en el régimen de ejecución del trabajo: constituyen indicios comunes de la misma la asistencia al centro de trabajo del empleador o al lugar de trabajo designado por éste y el sometimiento a un horario; también el desempeño personal del trabajo, compatible en determinados servicios con un régimen excepcional de suplencias o sustituciones; la inserción del trabajador en la organización de trabajo del empleador, que se encarga de programar su actividad y dirigirla; $y$, reverso de lo anterior, la ausencia de organización empresarial propia del trabajador.

Conforme a ello, el Juzgado de lo Social 39 de Madrid afirma que se ha acreditado en juicio que el demandante (i) no tenía jornada ni horario de trabajo ${ }^{13}$, no teniendo tampoco que justificar sus ausencias $^{14}$; (ii) tenía dominio completo de su actividad $^{15}$; (iii) gozaba de la capacidad de auto-organización ${ }^{16} y$, con ello, Glovo no ostentaba poder disciplinario alguno; (iv) asumía el riesgo y ventura

10 Resolución del Juzgado de lo Social 39 de Madrid recaída en la resolución 284/2018, del 3 de setiembre de 2018.

11 Segundo párrafo del Hecho Probado Tercero de la resolución 284/2018 del Juzgado de lo Social 39 de Madrid.

12 Fundamento de Derecho Tercero de la resolución 284/2018 del Juzgado de lo Social 39 de Madrid, que, citando a distintos pronunciamientos previos, define los indicios comunes de dependencia y ajenidad propios del vínculo laboral.

13 Numeral 2 del Fundamento de Derecho Quinto de la resolución 284/2018 expedida por el Juzgado de lo Social 39 de Madrid, que señala: “(...) el demandante no tenía jornada ni horario. Él decidía la franja horaria en la que deseaba trabajar, elegía los pedidos que le interesaban y rechazaba los que no quería, decidía con libertad la ruta a seguir hasta cada destino, no tenía obligación de comenzar o finalizar su jornada en una determinada hora, la Empresa no le imponía la clase o número de pedidos que tenía que hacer ni la ruta para llevarlos a cabo, el pedido se realizaba siguiendo las instrucciones dadas por el cliente final -no por GLOVO-, entrando el repartidor en contacto directo con el cliente una vez aceptado el pedido".

14 Numeral 5 del Fundamento de Derecho Quinto de la resolución 284/2018 expedida por el Juzgado de lo Social 39 de Madrid, que señala: "(...) no tenía que justificar ausencias, solo comunicarlas a la Empresa (...) no consta que se le pida una justificación de su ausencia o que se le rebaje la puntuación por ese motivo".

15 Numeral 2 del Fundamento de Derecho Quinto de la resolución 284/2018 expedida por el Juzgado de lo Social 39 de Madrid, que señala: "(...) p[udiendo] incluso desistir de un servicio previamente aceptado a mitad de ejecución sin sufrir por ello penalización alguna”.

16 Numeral 3 del Fundamento de Derecho Quinto de la resolución 284/2018 expedida por el Juzgado de lo Social 39 de Madrid, que señala: “(...) la demandada no tiene poder disciplinario sobre el actor más allá del desistimiento del propio contrato en el caso que de que no se realizaran servicios (elemento consustancial a cualquier contrato bilateral), y de una pequeña 'penalización' en su puntuación (...) la inexistencia de poder disciplinario es consecuencia de la capacidad de auto-organización del trabajador, no pudiendo la Empresa imponer sanciones por la falta de aceptación de pedidos, la forma de realización de los mismos o el abandono de un pedido previamente aceptado". Inclusive, a continuación, se indica de manera expresa que se acreditó en autos que el geo localizador GPS “(...) no era un instrumento de control de la Empresa, sino la forma de contabilizar el kilometraje para su posterior abono en la factura siguiente. No consta que se utilizara para controlar la ruta elegida por el actor en cada recado". 
en la prestación de servicios ${ }^{17}$, sin someterse a una estructura organizativa de la empresa; y, (v) aportaba las principales herramientas de trabajo.

Por su parte, el Juzgado de lo Social 17 de Madrid, mediante la Sentencia $12 / 2019^{18}$, reconoció el 11 de enero de 2019 la existencia de una relación de trabajador autónomo económicamente dependiente (trade) entre la parte demandante y Glovo. Para ello, al igual que en la sentencia antes detallada, valoró similares hechos probados a partir de los cuales concluyó que el proveedor de servicios de esta app goza de amplias libertades para el ejercicio de sus funciones, lo que lo califica como un trabajador autónomo. Conforme a ello se afirma que:

[...] se ha acreditado que el repartidor tiene total libertad para elegir los días en los que quiere ofrecer su servicio en GLOVO y la franja horaria para entregar los productos en cada jornada y para decidir sus jornadas de descanso. Asimismo, puede escoger si quiere prestar todos los servicios que le proponga la empresa (modalidad de asignación automática), aunque con la posibilidad de rechazar aquellos pedidos que no le interese realizar sin tener que justificar el motivo (pero debiendo comunicar e rechazo, para que el pedido sea asignado a otro repartidor) o bien aceptar uno a uno los pedidos que estén disponibles (modalidad de asignación manual). Incluso, una vez iniciado el servicio, el repartidor puede elegir desistir del pedido para realizar otro o finalizarlo. También tiene total libertad de organización y decisión a la hora de seleccionar la ruta desde la posición inicial hasta la dirección de recogida y hasta la dirección de entrega sin tener establecida una trayectoria ni un punto concreto de localización para el inicio del servicio, aceptando el repartidor un modelo de facturación por distancia lineal o real óptima $[. . .]^{19}$.

De forma contraria, el Juzgado de lo Social 33 de Madrid, mediante Sentencia 53/1920, reconoció el 11 de febrero de 2019 la existencia de una relación de naturaleza laboral entre la parte demandante y Glovo. Para ello, el Juzgado alegó la existencia de un cambio de paradigma en las relaciones de trabajo como consecuencia de la proliferación de la llamada tecnología de la información y comunicación (TIC), lo que a su entender vuelve necesario que los vínculos jurídicos que pudieran implicar cierta dependencia y, a su vez, hagan uso de TICs, sean evaluados de manera distinta, dejando de lado los conceptos que se valoraban previamente, bajo otro tipo de organización empresarial.

En este sentido, si bien es cierto que el trabajo autónomo (o trabajo por cuenta propia) y el trabajo dependiente (o trabajo por cuenta ajena) se distinguen porque únicamente en este último es posible identificar los elementos de ajenidad y dependencia. Se estima que la incidencia de las TIC es tan relevante que "[...] al momento de analizar si concurren las notas de ajenidad y dependencia y para lograr una adecuada calificación de la relación es preciso analizar los nuevos indicios que aparecen, antes inexistentes, y volver a valorar el peso definitorio de los indicios clásicos [...]"21.

Ciertamente, a decir del juzgado, la forma cómo se evalúa el trabajo ejecutado (i.e. una de las manifestaciones del ejercicio del poder de dirección del empleador) y la remuneración correspondiente han cambiado con el paso del tiempo, pues, gracias al uso de las TIC, ya no es necesario ejecutar control directo a través de otra persona ni resulta muy oneroso o excesivamente meticuloso poder conocer con exactitud el tiempo de actividad empleado por quien ejecuta los servicios, todo lo cual permite que las empresas estructuren modelos de negocio que pretenden únicamente reconocer el tiempo efectivo de trabajo y no la sola puesta a disposición de la fuerza laboral. Así, el juzgado afirma que:

[...] no es preciso el control directo por otra persona, el mando intermedio, ya que esta tarea puede encomendarse a sistemas de control automatizados sean cámaras, ordenadores, sistemas de geolocalización, etc. Y, además, las TIC permiten acceder a un gran volumen de información y a su tratamiento rápido y barato mediante la creación de los correspondientes algoritmos que a través de la ela-

17 Numeral 4 del Fundamento de Derecho Quinto de la Sentencia 284/2018 expedida por el Juzgado de lo Social 39 de Madrid, que señala: “(...) el repartidor asume el riesgo y ventura de cada pedido y responde del mismo frente al cliente, que también es quien puntúa su trabajo. No consta el sometimiento del trabajador a una estructura organizativa de la empresa, que sólo decide las tarifas con que abonará los servicios, el lugar de la prestación de los mismos y la herramienta a través de la cual oferta los 'recados' (APPP) siguiendo un programa informático que busca minimizar la suma de costes notas que son compatibles con la prestación de trabajo en régimen de autónomo (...)".

18 Resolución 12/2019 del Juzgado de lo Social 17 de Madrid. Sentencia expedida el 11 de enero de 2019.

19 Penúltimo párrafo del Segundo Fundamento de Derecho de la Sentencia 12/2019 expedida por el Juzgado de lo Social 17 de Madrid.

20 Resolución 53/19 del Juzgado de lo Social 33 de Madrid. Sentencia expedida el 11 de febrero de 2019.

21 Fundamento de Derecho Quinto de la resolución 53/19 expedida por el Juzgado de lo Social 33 de Madrid. 
boración de perfiles acceden al conocimiento detallado de cuándo, cómo, dónde y con qué resultado se ha trabajado. Todo ello a su vez permite vincular la retribución compensatoria del trabajo, no el tiempo en que el trabajador está a disposición sino el resultado final de su actividad $[\ldots]^{22}$.

Bajo esta nueva forma de valorar los elementos del vínculo jurídico, el juzgado encontró una serie de indicios $^{23}$ a partir de los cuales podría inferirse la existencia de un vínculo laboral entre los proveedores de servicio y Glovo, entre los cuales se destacan los siguientes:

a) El repartidor no interviene en la conformación del contenido del contrato lo que demuestra una posición de desigualdad entre las partes.

b) El contrato entre las partes regula rasgos que demuestran la sumisión del repartidor respecto de la empresa, entre otros, la impartición de criterios a seguir para la compra de productos y relaciones con el cliente final; el establecimiento de un límite máximo de minutos para la realización de un encargo; la prohibición de uso de distintivos corporativos propios o diferentes de los de la empresa; la prohibición de uso de la imagen corporativa en redes; el establecimiento de un listado de causas justificadas de resolución del contrato así como interrupciones de la actividad que se consideran justificadas; la prohibición de revelar secretos industriales.

c) El repartidor no establece o negocia su contraprestación por el servicio ejecutado pues la misma dependerá de los parámetros que la empresa establece para el reparto.

d) Aunque se afirma que el consumidor final y el repartidor entablan una relación directa, el pago que el primero efectúa se hace a través de la app lo que permite que la empresa descuente una comisión empresarial por sus servicios (de intermediación).

Al respecto, nos parece importante mencionar que, desde nuestra perspectiva, la mayoría de las situaciones calificadas como indicios de la existen- cia de un vínculo de naturaleza laboral no implican, necesariamente, que la misma se configure pues muchos pueden encontrarse en vínculos de naturaleza autónoma sin que ello conlleve a que nos encontremos ante una desnaturalización. Así, por ejemplo, no es extraño que cuando se negocia un contrato, cualquiera este sea, una de las partes ostente mayor poder para negociar e imponer sus condiciones. Ello debido a que la realidad es que alguna de ellas siempre tiene una posición más favorecida que la otra. De igual forma, el establecimiento de reglas para el uso de una marca no implica automáticamente el ejercicio de poder de subordinación, pues, al ser la marca un activo tan importante, el establecimiento de límites para su explotación de cara a terceros es necesario para salvaguardar una imagen en el mercado. En el mismo sentido, la mayoría -por no decir todos- los contratos de naturaleza civil, ya sea para la prestación de servicios personales o de outsourcing, contienen cláusulas que regulan las causales de terminación anticipada así como una prohibición de revelar secretos industriales. Este es un aspecto lógico pues se esperan reglas claras frente a posibles eventualidades que prefieren evitarse $y$, además, en el marco de un negocio en el que muchas veces se puede sacar ventaja de los competidores a partir del know how, lo más saludable es resguardar estas particularidades, evitando que sean difundidas por terceros que pudieran haberlas conocido. Nada de lo dicho nos parece un indicio de naturaleza laboral, sino que, por el contrario, se trata de situaciones que aparecen como consecuencia de la realidad o como una premisa ante la necesidad de garantizar las mejores condiciones para la explotación del principal activo del negocio.

Ahora bien, el principal razonamiento aportado por el juzgado radica, según vemos, en el desarrollo de una nueva forma de evaluar el ejercicio de funciones subordinadas y el tiempo de trabajo, ya que indica que, a partir del uso de las TIC en las relaciones laborales, se ha generado un nuevo sistema de trabajo basado en dos aspectos fundamentales ${ }^{24}$ : (i) la atomización de micro tareas sucesivas en función a la demanda, que perite que una misma relación contractual permanezca "hibernada" y se actualice con la asignación de cada una de ellas; y, (ii) la aparición de una facultad de conformidad del prestador a cada encomienda que se le ofrezca, manifestando así su voluntad de prestar servicios.

22 Antepenúltimo, penúltimo y último párrafos del Fundamento de Derecho Segundo de la Sentencia 53/19 expedida por el Juzgado de lo Social 33 de Madrid.

23 Fundamentos de Derecho Sétimo, Octavo y Noveno de la Sentencia 53/19 expedida por el Juzgado de lo Social 33 de Madrid.

24 Fundamento de Derecho Décimo de la Sentencia 53/19 expedida por el Juzgado de lo Social 33 de Madrid. 
Siguiendo la misma lógica, el juzgado valoró que "[...] el éxito de este tipo de plataformas se debe al soporte técnico proporcionado por las TIC [...] y a la explotación de la marca [...] que se publicita en redes sociales a través de los buscadores tipo Google, sitio al que acuden los clientes cuando necesitan la compra y entrega de los productos que la demandada suministra [...]"25. Así, a partir de esta afirmación, se concluye que los medios materiales aportados por los proveedores de los servicios (i.e. medios de transporte y de telefonía) tienen un escaso valor si se comparan con lo que ostenta la empresa (no solo el algoritmo y la $a p p$, sino también la marca en el mercado), por lo que, si estos últimos no existieran, sería inviable que los repartidores pudieran llevar a cabo por sí solos la misma actividad económica, lo que a criterio del juzgado confirma que existe una prestación que se ejecuta bajo la característica de ajenidad del servicio ${ }^{26}$.

Finalmente, en el mismo sentido que esta última sentencia comentada, el Juzgado de lo Social 1 de Madrid, mediante Sentencia 134/1927, reconoció con fecha 4 de abril de 2019 la existencia de una relación de naturaleza laboral entre el demandante y Glovo. Para ello, además de basarse en similar jurisprudencia, el juzgado valoró que el hecho que la empresa asumía íntegramente el pago de una póliza de seguro que cubría amplios riesgos en beneficio de los proveedores, elemento que no se tuvo a revisión en los pronunciamientos previos.

\section{Pronunciamientos referidos a Deliveroo}

Deliveroo es una empresa fundada en el 2013 en Londres y se encarga de colocar a disposición de distintos usuarios una serie de personas que les brindan apoyo haciendo repartos principalmente de comida; esta empresa es la titular de una app que ha recibido los pronunciamientos más recientes de la justicia española en los que se declara la existencia de un vínculo de naturaleza laboral entre la plataforma y los proveedores de servicios.

Así, respecto de ella, mediante Sentencia 244/2018 ${ }^{28}$, el Juzgado de lo Social 6 de Valencia reconoció con fecha 1 de junio de 2018 la existencia de una relación de naturaleza laboral entre la parte demandante y Deliveroo. Para ello, el juzgado señala que en el vínculo suscrito entre los repartidores y la empresa se pueden identificar los datos característicos de ajenidad y dependencia, presentando esta relación jurídica "[...] rasgos que solo son concebibles en el trabajo dependiente y por cuenta ajena [...]"29. Conforme a ello, se identifican los siguientes elementos que acreditarían tanto dependencia como ajenidad en la prestación de servicios ${ }^{30}$ :

a) El repartidor trabajaba siguiendo las instrucciones de la empresa y bajo las condiciones fijadas unilateralmente por ella.

b) La empresa decidía la zona en la que se desempeñaban las funciones.

c) Aunque el repartidor ofertaba a la empresa las franjas horarias en las que quería prestar servicios, éstas debían estar dentro del horario previamente establecido por aquélla, quien decidía el horario semanalmente.

d) La empresa daba instrucciones concretas a los repartidores sobre la forma en que el servicio se debía llevar a cabo, fijando tiempos y normas de comportamiento que aquéllos debían cumplir.

e) La empresa fijaba un lugar de trabajo que servía de punto de partida: los repartidores debían acudir al centroide para que la empresa les asignara servicios a través de la $a p p$, debiendo retornar al mismo al finalizar cada servicio.

f) La empresa mantiene geo localizado al repartidor de forma permanente, pudiendo exigir explicaciones sobre la ejecución del servicio en cualquier momento, llevando control del tiempo de cada reparto y decidiendo permanentemente sobre la efectiva asignación de repartos.

g) Una vez que la empresa asignaba un turno de reparto, en caso el repartidor no pudiese cumplirlo, debía encontrar a otro repartidor que lo sustituyera, debiendo obtener la autorización de la empresa para proceder con el cambio.

25 Fundamento de Derecho Duodécimo de la resolución 53/19 expedida por el Juzgado de lo Social 33 de Madrid.

26 Fundamento de Derecho Décimo Tercero de la resolución 53/19 expedida por el Juzgado de lo Social 33 de Madrid.

27 Resolución 134/19 del Juzgado de lo Social 1 de Madrid. Sentencia expedida el 04 de abril de 2019.

28 Resolución 244/2018 del Juzgado de lo Social 6 de Valencia. Sentencia expedida el 01 de junio de 2018.

29 Fundamento de Derecho Segundo de la resolución 244/2018 expedida por el Juzgado de lo Social 6 de Valencia.

30 Fundamento de Derecho Segundo de la resolución 244/2018 expedida por el Juzgado de lo Social 6 de Valencia. 
h) El repartidor carece de libertad, dentro de la franja horaria elegida, para rechazar los pedidos -se acreditó que la empresa manifestó que tanto el rechazo de ofertas como la falta de disponibilidad reiteradas dieron lugar a la extinción del vínculo entre las partes-.

i) Para suspender temporalmente la prestación de servicios, el repartidor debía enviar una comunicación a la empresa con dos semanas de antelación.

j) Aun cuando el repartidor aporta sus propios medios de trabajo (bicicleta y teléfono móvil), carece de organización empresarial y es la empresa la que a través de la app organiza su actividad.

k) La empresa decidía el precio de los servicios ejecutados por el repartidor, quien los percibía con independencia del cobro que ejecutara aquella.

I) La empresa establecía las condiciones de los restaurantes adheridos y de los clientes a los que se les prestaba servicios, desconociendo el repartidor con antelación a la asignación la identidad de todos ellos.

m) La empresa reconoce que los repartidores son "la imagen de la compañía de cara al cliente".

En este sentido, mediante Sentencia 193/201931, el Juzgado de lo Social 31 de Barcelona reconoció con fecha 11 de junio de 2019 la existencia de una relación de naturaleza laboral entre la parte demandante y Deliveroo. Para ello, se valoraron una serie de elementos fácticos que no se pudieron corroborar en los demás casos materia de comentario. Así, se verificó que la empresa (i) otorgaba materiales de trabajo - un soporte para llevar el teléfono móvil en la bicicleta o motocicleta, una batería externa, una caja de transporte o mochila con el logotipo de Deliveroo, así como por un tiempo pantalones, chaquetas y gorra con el mismo logotipo-32; (ii) imponía como una obligación que, durante un primer periodo de prestación de servicios, los candidatos a repartidor realizaran algunas horas de prueba acompañados de un repartidor con experiencia, al que denominaban trainer ${ }^{33}$; y, (iii) fiscalizaba los servicios ejecutados por los repartidores, generando a partir de esta supervisión "métrica" que tomaban en consideración el porcentaje de rechazo, la asistencia a las franjas para las que el repartidor había mostrado disponibilidad, la velocidad media de tiempo transcurrido entre la oferta de pedido y su entrega y la disponibilidad del repartidor en franjas de alta demanda, a partir de todo lo cual se asignaba un posicionamiento de prioridad para la siguiente elección de las franjas horarias ${ }^{34}$.

De conformidad con este último pronunciamiento, los hechos probados revelaron que los repartidores prestaban sus servicios plenamente integrados en el ámbito de organización y dirección de la empresa y que la alegada libertad de elección de los mismos no correspondía con la realidad en modo alguno ${ }^{35}$. Así, por ejemplo, se desarrolla algún comentario respecto de la forma de comunicación que tiene la empresa respecto de los repartidores, a quienes se dirige en términos imperativos -a veces, en mayúsculas o negritas-, insistentes y de advertencia, todo lo cual, a criterio del juzgado, no sería compatible con las facultades de auto organización y autonomía de un independiente.

No obstante, en esta sentencia se desarrolla con mayor detalle el concepto de la ajenidad en la marca, cuestión que es atribuida al profesor Adrián Todolí y que propone que es innegable que los repartidores actúan en el mercado amparados por una marca y que "[...] con su prestación de servicios [...] no ponen en juego, ni para bien ni para mal, otra cosa que la [propia] marca [...] siéndoles por tanto totalmente ajeno el impacto de su actividad ya que el mismo repercute en [aquella] [...]"36.

Así, se propone que el elemento productivo estrella no es ninguno de los proporcionados por los repartidores (ni la bicicleta, ni el teléfono, ni el propio repartidor), sino una marca que utiliza la $a p p$ para promocionar sus servicios, sin el cual "[...] no ha[bría] negocio posible" ${ }^{37}$ y que, pese a ello, los repartidores eran absolutamente ajenos a la misma, siendo su única vinculación la de promoción pues “[...] lleva[n] su logotipo en la caja de reparto y s[on] su cara visible para los clientes en el momento de la entrega $[\ldots]^{\prime \prime 38}$.

\footnotetext{
31 Resolución 193/2019 del Juzgado de lo Social 31 de Barcelona. Sentencia expedida el 11 de junio de 2019.

32 Octavo y Noveno Hechos Probados de la resolución 193/2019 expedida por el Juzgado de lo Social 31 de Barcelona.

33 Décimo Tercer Hecho Probado de la resolución 193/2019 expedida por el Juzgado de lo Social 31 de Barcelona.

34 Vigésimo Cuarto Hecho Probado de la resolución 193/2019 expedida por el Juzgado de lo Social 31 de Barcelona.

35 Fundamento de Derecho Tercero de la resolución 193/2019 expedida por el Juzgado de lo Social 31 de Barcelona

36 Fundamento de Derecho Tercero de la resolución 193/2019.

37 Fundamento de Derecho Tercero de la resolución 193/2019.

38 Fundamento de Derecho Tercero de la resolución 193/2019.
} 


\section{Comentarios respecto de los indicios de laboralidad contenidos en los distintos pro- nunciamientos judiciales}

A partir de la revisión de la jurisprudencia antes detallada, encontramos que los juzgados españoles no solo pretenden analizar los vínculos jurídicos desarrollados en el interior de la on-demand economy desde los conceptos y elementos tradicionales del Derecho del Trabajo, sino que, además, atendiendo a la nueva dinámica existente en el desarrollo de los mismos, han aceptado conceptos novedosos como el de ajenidad de la marca con miras a encontrar elementos que adapten las formas de subordinación a una realidad cada vez menos presencial y más informática.

Por nuestra parte, coincidimos con los pronunciamientos judiciales cuando señalan que, desde que se evidencia subordinación en el vínculo jurídico (i.e. cuando la empresa ejerce los poderes de dirección, fiscalización y sanción), nos encontraremos ante una relación laboral. En efecto, la ausencia de un trato directo, inmediato o físicamente cercano con los proveedores no significará de forma alguna que los mismos no puedan ni se encuentren vigilados o fiscalizados por la empresa ni que ésta, alegando la necesidad de alimentar su algoritmo, pueda ejercer de forma indirecta un control respecto del desarrollo de la actividad.

Así, por ejemplo, en los casos en los que se pudiera establecer que las empresas establecen rutas obligatorias a los conductores o repartidores, nos encontraríamos ante una situación en la que se están emitiendo directivas específicas sobre cómo se debe prestar el servicio, potestad que no es compatible con una contratación de una actividad supuestamente autónoma. En efecto, el establecimiento de una ruta no es una facilidad que la empresa brinde al proveedor de servicio como una mera recomendación que busca simplificar el servicio; se trata, en realidad, de una orden que estos deben cumplir, limitando así cualquier margen de decisión que pudieran tener los proveedores de servicio.

En este caso, si la dinámica permite que la empresa no solo asigne al cliente sino también indique al proveedor qué ruta seguir, entendemos que las facultades que ostenta la empresa convertirían al proveedor en un simple ejecutor, incorporándolo dentro de una estructura empresarial y convirtiendo su vínculo en uno de naturaleza subordinada y, por ende, propio del Derecho del Trabajo.

Ahora bien, en lo que se refiere a la provisión de los medios o herramientas de trabajo, consideramos que si bien la app o plataforma representa un elemento mucho más complejo y costoso que la sola puesta a disposición del medio de transporte (sea auto, bicicleta o cualquiera de menor dimensión) y el teléfono móvil, lo cierto es que sin la concurrencia de cualquiera de ellos, la actividad y los servicios ofertados a través de estas empresas no podrían ejecutarse, siendo por tanto todos ellos importantes y consustanciales al negocio. Ciertamente, de nada serviría haber desarrollado un aplicativo como el de Uber si, finalmente, la misma empresa tuviera la necesidad y obligación de facilitarse vehículos para la ejecución de los servicios. Sumado a ello, encontramos que el hecho de que los proveedores asuman, por ejemplo, costos inherentes al mismo (e.g., paguen sus licencias y multas, se encarguen de limpiar los vehículos, paguen por el combustible que consumen, entre otros) es un elemento que evidencia que éstos ejecutan sus servicios a su cuenta y riesgo.

Debido a los anteriormente expuesto, insistimos en que para el éxito de este tipo de negocios tanto la plataforma como el servicio y los medios con los que se presta el mismo son sumamente importantes y dependientes entre sí: si bien es cierto que el desarrollo de una buena plataforma digital generará la proliferación de su uso, de nada serviría tener un gran sistema operativo si las personas que ofrecen sus servicios a través del mismo no cumpliesen estándares de calidad mínimos acorde al mercado, pues esto desincentivaría el uso de la tecnología. Por ello, es en este contexto que se puede entender el establecimiento de parámetros o requisitos para la ejecución de los servicios en el marco de determinada plataforma, los cuales podrían tener por finalidad garantizar ciertos estándares mas no subordinar la actividad.

Muchas veces, por ejemplo, las empresas titulares de las apps vinculadas a servicios de taxi requieren que sus proveedores cuenten con vehículos de determinados colores, marcas o años de antigüedad, o que las personas que ejecutan el servicio se encuentren prohibidas de vestir de determinada manera mientras se encuentren en actividad (e.g., se prohíbe el uso de camisetas sin manga o pantalones cortos o sandalias). Estos elementos han sido alegados como supuestas demostraciones del ejercicio del poder de dirección por parte de las empresas, algo que no compartimos en tanto resulta lógico que una empresa cuyas ganancias se encuentran estrechamente vinculadas con la imagen y calidad de servicio que los prestadores ofrecen al mercado se preocupe por establecer parámetros mínimos de cumplimiento de cara a ofrecer una conexión con servicios dirigidos a ciertos mercados o con determinadas características.

A nuestro parecer, el establecimiento de requisitos mínimos no representa la impartición de un orden 
ni el control de la forma cómo debe ejecutarse la prestación, pues, en realidad, únicamente garantiza que no se exceda un límite, mas no obliga a cumplir preceptos específicos e inflexibles: si se prohíbe, por ejemplo, el uso de camisetas sin mangas y pantalones cortos a los conductores de un vehículo, ello no significa que se les imponga el uso de un uniforme, sino que los mismos siguen manteniendo la posibilidad de elegir qué vestir dentro de varias posibilidades, tales como pantalones jean, pantalones de drill, traje, camisa, polo con cuello, polo sin cuello pero con mangas, etc; de igual forma, si no se aceptan vehículos con una antigüedad mayor a determinado número de años, ello no significa que se obligue a los proveedores de servicios a adquirir vehículos nuevos sino que se les permite emplear distintas alternativas siempre que calcen en un margen previamente determinado.

Habíamos señalado previamente que el negocio alegado por las empresas dueñas de las apps, a través de las cuales se ofrecen distintos servicios consiste en ser mediadores que facilitan el encuentro entre la oferta y la demanda. Conforme a ello, la prestación que estas empresas ofrecen a sus usuarios -quienes son tanto los proveedores como los consumidores- es el de contactarlas, lo que se logra incluyendo a un conjunto de personas naturales en su base de datos de proveedores y poniéndola a disposición de los consumidores que descargan la $a p p$, todo lo cual tiene por finalidad facilitar la información del mercado y agilizar la celebración de un contrato entre ambas partes. Tal y como se presenta, podría decirse que las plataformas cumplen la función de los antiguos tablones de anuncios o agencias de empleo en los que personas independientes publicaban su disponibilidad para prestar determinados servicios y quienes los requerían utilizaban esta información para seleccionar entre los ofertantes, todo lo cual generaba una comisión para el dueño del tablón o de la agencia de empleos por haber sido quien les facilitó el contacto.

Las apps, sin embargo, valiéndose de las funcionalidades que les proporcionan las herramientas de programación e inteligencia de negocios (i.e. de su algoritmo), van más allá del hecho de ser simples depositarias de oferta a la espera de la demanda; en efecto, el servicio que brindan a los consumidores muchas veces les facilita la toma de decisiones al mostrarles una serie de elementos que pueden influir en ella. Así, tal y como se ha expuesto en los hechos de las sentencias revisadas, a través de la forma cómo se haya programado el algoritmo que rige la plataforma, esta puede presentar a los proveedores en un orden predeterminado que responda a alguna característica deseada, por ejemplo, en función al puntaje que le han otorgado otros usuarios en base al nivel de satisfacción que les dejó su prestación de servicios; en función a la cercanía geográfica de los proveedores respecto de la ubicación del consumidor; atendiendo al número de quejas presentadas por consumidores anteriores, entre otros.

La plataforma podría, inclusive, sustituir la toma de decisión del consumidor y elegir directamente lo que en función a ciertos parámetros objetivos predeterminados se considera lo más adecuado (para lo cual se recurre a criterios objetivos de eficiencia y menor costo e inversión de recursos). Todas estas facilidades de información y de clasificación de la misma representan, evidentemente, un servicio prestado por la empresa en favor de sus usuarios y, como tal, implicará el pago de una tarifa tanto de los proveedores que se exponen en esta herramienta como de los consumidores que se benefician del procesamiento de la información. Sobre el particular, conviene insistir en que, al depender las ganancias de la empresa del número de servicios que se concreten a través de su uso, la lógica económica nos lleva a pensar que esta siempre estará interesada en que los consumidores se encuentren satisfechos con los servicios contratados pues, en la medida que esto suceda, la app continuará en uso o inclusive será recomendado a terceros, todo lo cual le permitirá mayores ingresos.

Desde nuestra perspectiva, esta lógica mercantil resultará plenamente válida siempre que en sus actuaciones y en la reserva de sus facultades, la empresa dueña de la app no se irrogue la posibilidad de ejercer comportamientos que subordinen a los prestadores, algo que indefectiblemente sucederá si -en el marco de sus potestades- la empresa tiene la posibilidad de subordinar al proveedor, i.e. ejercer los poderes de control y sanción respecto del mismo.

Pero, ¿cuándo podría entenderse que la empresa incurre en este ejercicio? Desde nuestro punto de vista, el poder de control se materializará cuando la fiscalización efectuada por la empresa denote injerencia y manejo de la forma de ejecutar las actividades del proveedor. De igual forma, se evidenciará el ejercicio de un poder sancionador cuando la finalidad de una decisión adoptada por la empresa sea reprender o castigar al proveedor como consecuencia de la detección de determinada conducta, lo que sucedería, por ejemplo, si se les suspendiera el derecho a seguir utilizando la app o si se procediera a modificar las tarifas o porcentajes que les corresponde por los servicios ejecutados como consecuencia de la acumulación de determinado número de quejas presentadas por los consumidores o, inclusive, si ello sucediese 
cuando no se llegue a un número mínimo de servicios prestados dentro de un determinado lapso de tiempo.

En estos casos, la actividad de la empresa no se estaría limitando a la de ser un simple espectador que cumplió las veces de enlace o contacto entre las partes de un contrato, sino que estaría interviniendo activamente en la ejecución del servicio con la finalidad de controlarlo o dirigirlo, lo que excede sus facultades como contraparte en el marco de un contrato de servicios independientes o autónomos. Al respecto, podría alegarse que la empresa necesita generar mecanismos que: (i) desalienten a los proveedores a incurrir en comportamientos que generen molestias e insatisfacción por parte de los consumidores; o, (ii) tengan por finalidad asegurar una determinada constancia en el uso de la app para ofrecer sus servicios; por lo que, para ello, lo más efectivo podría resultar ejecutar una medida que les represente una afectación económica.

A nuestro entender, el que las plataformas exhiban un sistema de puntuación de los proveedores como parte de su estructura representa de por sí un elemento disuasivo frente a las conductas que se desea evitar porque a través del mismo tanto los consumidores como el propio algoritmo que rige el sistema podrían discriminar entre quienes resultan más adecuados para ofrecer el servicio requerido, prefiriendo la elección de quienes: (i) tuvieren una mayor puntuación generada por el nivel de satisfacción de usuarios previos; o, (ii) fueren reconocidos por la propia aplicación como proveedores habituales del servicio. Esto, insistimos, no es más que una herramienta provista por el sistema de la app que contribuye a la toma de decisión más no la sustituye, manteniendo dentro de su oferta a los proveedores con baja puntuación para que sean los usuarios quienes finalmente decidan si contratan o no con ellos.

¿Significará esta posición que la empresa se encuentra impedida de aplicar la "expulsión" de un proveedor de su sistema ante determinadas ocurrencias? De ninguna manera. La resolución del vínculo contractual existente entre la empresa y el proveedor que ofrece sus servicios a través de la app es posible y aplicará como consecuencia de la ocurrencia de algún supuesto que haga imposible la consecución de la relación como, podría ser, la toma de conocimiento de una vinculación del proveedor con algún ilícito. Recordemos, pues, que la lógica económica de este sistema lleva implícito el incremento de las ganancias empresariales a partir de la mejor imagen y confianza que genere la empresa en el mercado, lo que determina que el nombre de la empresa pueda verse perjudicado en función de con quién se relaciona y a quiénes mantiene entre sus proveedores, por lo que dejar de relacionarse con quien pueda opacar su imagen en el mercado responde no a un deseo de sanción sino a una necesidad estratégica.

Algo similar sucede, por ejemplo, en las relaciones interpersonales en las que sin ganar comisión alguna recomendamos a una persona para la ejecución de algún servicio, e.g. a un pintor que previamente hubiera pintado nuestra casa, a un amigo que sabemos ostenta determinada especialidad en el Derecho, a una persona que se encarga de hacer trámites, etc. En este contexto, si en cierto momento se nos informa que alguna de estas personas que han sido recomendadas por nosotros, ha procedido de forma tal que hubiere perjudicado a quien se la recomendamos (e.g., ya sea porque incumplió los plazos acordados o porque no concluyó el servicio y, pese a ello, se apropió del íntegro de los honorarios como si el mismo se hubiera ejecutado totalmente), la lógica nos lleva a pensar que no volveremos a recomendar a esta persona, pues nuestra imagen, nombre o reputación se pueden ver manchados como consecuencia del mal proceder de este tercero. Según entendemos, el mismo razonamiento debiera aplicarse ante la necesidad de una empresa de desvincularse de quienes pudieran dañar su imagen, máxime si se trata de una afectación que impactará económicamente en el desarrollo del negocio. Recordemos, pues, que en la práctica la percepción que tenga el mercado respecto de la idoneidad del servicio prestado por los proveedores ofrecidos en una plataforma le representa publicidad, la que no solo puede significar una carga positiva sino también negativa y que, por ello, es esta última consecuencia la que se debe evitar.

En todo caso, nuestra recomendación es, finalmente, evaluar la forma cómo se desarrolla cada actividad empresarial y verificar tanto en teoría como en la práctica, cuáles son las facultades y prerrogativas de cada parte; así, si lo que se quiere es mantener fuera del ámbito laboral las relaciones jurídicas evaluadas, lo que se debe descartar es la existencia de elementos que denoten subordinación en su desarrollo.

\section{CONCLUSIONES}

a) El uso de la tecnología ha conducido a una amplia descentralización productiva, que permite a las empresas de la on-demand economy facilitar la prestación de un servicio que declaran no ejecutar directamente.

b) El principal cuestionamiento que se efectúa a la economía basada en la prestación de 
servicios a través de plataformas digitales se sitúa en la supuesta simulación de vínculos jurídicos de naturaleza independiente que ésta generaría, algo que no es privativo de este tipo de actividad comercial.

c) A pesar de los beneficios que pueden generarse a partir de las iniciativas que surgen en el marco de la on-demand economy, las ventajas competitivas del nuevo modelo de negocios deben cimentarse en una mejor organización o una mayor productividad y de ninguna manera en el incumplimiento de la regulación vigente en cada jurisdicción, Esto incluye, además de la regulación laboral, el derecho regulatorio en los casos corresponda.

d) En nuestra opinión, en gran medida, los problemas que se pretende vincular con este tipo de economías se generan, en realidad, como consecuencia de problemas sociales: a medida que los salarios han disminuido en el empleo tradicional, los trabajadores han necesitado complementar sus ingresos con formas alternativas de trabajo, en este caso, a través de ocupaciones flexibles donde pueden prestar servicios solo en su tiempo libre. Pese a ello, la alegada flexibilidad no siempre representa una ventaja pues muchos trabajadores, que muchas veces prestan servicios en jornada completa para un empleador durante el día, optan por sumar un mayor número de horas de ocupación para prestar servicios bajo la on-demand economy. Por lo tanto, podríamos afirmar que, en muchos casos, las personas que realizan servicios bajo este tipo de economía no están eligiendo libremente esta tarea, sino que los bajos salarios los obligan a involucrarse en esta modalidad de vínculo jurídico. A ello, convendría añadir a todas aquellas personas que, pese a aspirar a ocupaciones propias de la economía tradicional, optan por prestar servicios a través de aplicativos por la ausencia de oferta de empleo formal en el mercado tradicional.

e) Es recomendable que las empresas que desarrollan sus negocios bajo la on-demand economy estructuren los mismos evitando incurrir en acciones que evidencien la existencia de un vínculo subordinado (por ejemplo, a partir del establecimiento de objetivos mínimos o la imposición de distintas formas de sanción), limitándose a prestar servicios comerciales como intermediarios. En el mismo sentido, es importante considerar que la libertad en la determinación del tiempo de trabajo y la elección del servicio a ejecutar, así como el uso de materiales o medios de producción propios, son ejemplos de un claro debilitamiento de los indicios clásicos de laboralidad.

f) Reciente jurisprudencia española ha validado teorías doctrinarias que proponen conceptos nuevos como el de ajenidad en la marca, así como la existencia de nuevos indicios que favorecerían la identificación de un vínculo de naturaleza laboral. Por nuestra parte, aplaudimos este afán de actualizar los conceptos de nuestra disciplina y abogamos porque los mismos sigan siendo debatidos, ojalá, bajo una perspectiva interdisciplinaria.

\section{REFERENCIAS}

EUR-Lex (s.f. a). "Reafirmación de la libre circulación de trabajadores: derechos y avances importantes". Disponible en: https://eurlex.europa.eu/legal-content/es/ALL/?uri= CELEX:52010DC0373

(s.f. b). "Una Agenda Europea para la economía colaborativa". Disponible en: https:// eur-lex.europa.eu/legal-content/ES/TXT/?uri= CELEX:52016DC0356

Gauthier Guazzoni, G. (2016) Impacto de las nuevas tecnologías en el mundo del trabajo. Disponible en: https://adriantodoli.com/wpcontent/uploads/2016/10/impacto-de-lasnuevas-tecnologc3adas-en-el-mundo-deltrabajo.pdf

Instituto Nacional de Estadística (25 de julio de 2019). "Encuesta de población activa (EPA)-Trimestre 2/2019". Recuperado de: https://www. ine.es/dyngs/INEbase/es/operacion.htm?c=Es tadistica_C\&cid=1254736176918\&menu=ultiD atos\&idp=1254735976595

Mercader Uguina, J.R. (11 de julio de 2017). “EI nuevo modelo de trabajo autónomo en la prestación de servicios a través de plataformas digitales". En: Diario La Ley. Disponible en: http://diariolaley.laley.es/Content/Documento.aspx? params $=\mathrm{H} 4$ sIAAAAAAAEAMtMSbF1C TEAAiNTQyMzQ7Wy1KLizPw8WyMDQ3MDc0 MjAGCFBL4hAAAAWKE

Neves Mujica, J. (2012). Introducción al derecho del trabajo. Segunda Edición. Lima: ARA Editores.

Todolí Signes, A. (2015). El Contrato de Trabajo en el s. XXI: La Economía Colaborativa, On-Demand Economy, Crowdsorcing, Uber Economy 
y otras formas de descentralización productiva que atomizan el mercado de trabajo. En: El trabajo en la era de la economía colaborativa. Valencia: Tirant lo Blanch.

Urrutia, C. (02 de julio de 2019). “España y Grecia lideran el desempleo con tasas que duplican la media de la UE". En: El Mundo. Disponible en: https://www.elmundo.es/economia/2019/07/ 02/5d1a4b2bfdddff94288b4633.html

\section{LEGISLACIÓN, JURISPRUDENCIA Y OTROS DOCU- MENTOS LEGALES}

Caso 2202551/2015 \& Otros (Uber BV v. Aslam), del Tribunal del Trabajo de Londres. Sentencia del 28 de octubre de 2016.

Caso C-434/15 (Asociación Profesional Élite Taxi contra Uber Systems Spain, SL), del Tribunal de Justicia de la Unión Europea. Sentencia del 20 de diciembre de 2017.

Ley 20/2007. Estatuto del trabajo autónomo. En: Boletín Oficial del Estado, 12 de julio de 2007.
Resolución 244/2018 del Juzgado de lo Social 6 de Valencia. Sentencia expedida el 01 de junio de 2018.

Resolución 284/2018, del Juzgado de lo Social 39 de Madrid. Sentencia del 03 de septiembre de 2018.

Resolución 12/2019 del Juzgado de lo Social 17 de Madrid. Sentencia expedida el 11 de enero de 2019.

Resolución 53/19 del Juzgado de lo Social 33 de Madrid. Sentencia expedida el 11 de febrero de 2019.

Resolución 134/19 del Juzgado de lo Social 1 de Madrid. Sentencia expedida el 04 de abril de 2019.

Resolución 193/2019 del Juzgado de lo Social 31 de Barcelona. Sentencia expedida el 11 de junio de 2019.

Sentencia 9197-2006-AA-TC, de Tribunal Constitucional del Perú. Expedida el 06 de noviembre de 2007. 